\title{
La paradoja de la familia doblemente recluida: clínica de una pandemia en Colombia
}

\author{
Norman Darío Moreno-Carmona \\ Doctor en Investigación Psicológica. Psicólogo \\ Universidad de San Buenaventura. Medellín, Colombia \\ https://orcid.org/0000-0002-8216-2569・norman.moreno@usbmed.edu.co
}

\author{
Juan José Cleves-Valencia \\ Magister en Psicología. Psicólogo \\ Universidad del Valle. Cali, Colombia \\ https:/ / orcid.org/0000-0002-3187-8137• juan.cleves@correounivalle.edu.co
}

Resumen

Este artículo de reflexión deriva de la investigación La familia colombiana actual y su relación con problemas internalizados y externalizados en los adolescentes. Su propósito es analizar las conclusiones de dicho estudio a propósito de la forma de educación impersonal de los hijos en la familia moderna, a partir de una lectura clínica de la Pandemia del COVID-19. Al develar la dinámica social que ha configurado los arreglos familiares y que puede comprenderse desde el devenir de la institución social del empleo y la escuela, se interroga de manera crítica la aparente inexorabilidad de la ausencia paterna en la familia, pero se resalta la paradoja de una nueva dinámica familiar en la que las medidas de confinamiento no necesariamente dan lugar al favorecimiento de mejores procesos de crianza o de interacciones paternofiliales.

Palabras clave: Clínica; Pandemia; Crianza; Familia; Reclusión; COVID-19.

Recibido: 30/07/2021 | Aprobado: 24/09/2021 | Publicado: 01/01/2022

(1) (2) Esta obra está bajo una Licencia Creative Commons Atribución-NoComercialCompartirIgual 4.0 Internacional.

Financiación o proveniencia del artículo: Artículo de reflexión resultado de la investigación La familia colombiana actual y su relación con problemas internalizados y externalizados en los adolescentes, realizada en el año 2018, financiada por la Universidad Católica Luis Amigó y la Universidad Cooperativa de Colombia.

\section{¿Cómo citar este artículo? / How to quote this article?}

Moreno-Carmona, N. D., y Cleves-Valencia, J. J. (2022). La paradoja de la familia doblemente recluida: clínica de una pandemia en Colombia. Prospectiva. Revista de Trabajo Social e intervención social, (33), 171185. doi: $10.25100 /$ prts.v0i33.10842. 
Moreno-Carmona y Cleves-Valencia

\title{
Colombian Families, Twice Secluded: A clinical Approach of the Pandemic
}

\begin{abstract}
The following reflection is derived from a research study titled The Current Colombian Family and its Relationship with Internalized and Externalized Problems in Adolescents. Its purpose is to analyse the conclusions of the referred study regarding the impersonal education of children in the modern family, based on a clinical approach of the COVID-19 Pandemic. Upon unraveling the social dynamics that have configured family arrangements -which are understood from the evolution of employment and school as social institutionsthe apparent inexorability of paternal absence in the family is questioned, and at the same time a paradox is highlighted -a new family dynamic in which confinement does not necessarily lead to the favoring of better parenting processes or parental-filial interactions.

Keywords: Clinic; Pandemic; Upbringing; Family; Reclusion; COVID-19.

Sumario: 1. Introducción, 2. Metodología, 3. Hallazgos, 3.1 Clínica de una pandemia, 3.2 Una lectura clínico social desde la dimensión del empleo, 3.3 Una lectura clínico social desde la dimensión de la escuela, 3.4 La crianza confinada, 4. Conclusiones, 5. Referencias bibliográficas.
\end{abstract}




\section{Introducción}

Este artículo de reflexión se deriva de la investigación La familia colombiana actual y su relación con problemas internalizados y externalizados en los adolescentes, y tiene como propósito analizar las conclusiones de dicho estudio a partir de lo que denominamos una Clínica de la Pandemia, en la que se plantean algunos aspectos de la dinámica familiar que es necesario replantear como posibilidades, a lo mejor impensables antes de la aparición del COVID-19. En una de sus conclusiones, la investigación refiere que "la educación de las familias modernas orienta en forma impersonal a sus hijos, sustentada en la necesidad del trabajo, la promoción académica y profesional de ambos padres o los proyectos personales de vida que no necesariamente involucran a los hijos" (Moreno-Carmona, 2018, p. 194), asunto que, a primera vista, se plantea como irreversible ante la imposición del modelo social y económico contemporáneo.

Entre otras variables, es la falta de dedicación de tiempo de los padres la que parece tornarse decisiva como factor de riesgo ante la presencia de problemas internalizados y externalizados en los adolescentes; si bien, no necesariamente se trata de la cantidad, sino de la calidad de dicho acompañamiento, al plantear que es algo "que ya la familia no ofrece, no puede ofrecer y no va a ofrecer" (Moreno-Carmona, 2018, p. 203). Además, el sugerir la necesidad de repensar el papel de la escuela y de otros referentes alternos a la familia en los procesos de crianza y socialización de las nuevas generaciones, deja entrever una supuesta e inapelable ausencia de los padres.

Pues bien, la llegada al mundo de una pandemia como el COVID-19 ha demostrado que aún las estructuras aparentemente más sólidas y estables del entramado social, como la economía, el trabajo, la escuela, el transporte, la producción y la familia pueden sufrir cambios en sus lógicas y dinámicas. Las posibles consecuencias que dichos cambios puedan generar en las familias son las que nos ocupan en esta reflexión.

No se trata aquí de acentuar posturas valorativas en términos de pérdidas (de vidas, de trabajos, de producción...), sino de tratar de entender su efecto en la sociedad, la familia y las personas, donde, paradójicamente, parecieran estar apareciendo resultantes positivas inesperadas: descontaminación, desaceleración, trabajo desde casa, reencuentro familiar, mayor participación de los padres en la crianza y en los procesos educativos de sus hijos y aprovechamiento de la virtualidad para la educación. 


\section{Metodología}

El diseño de la investigación empírica fue no-experimental, transversal, correlacional, de muestras independientes y multivariado, y se llevó a cabo en el escenario natural. Se trató de un estudio con una medición que buscó explorar la realidad familiar actual colombiana y algunos factores familiares y psicosociales asociados a problemáticas en la adolescencia. Se obtuvo una muestra con 968 adolescentes de 12 ciudades del país, de los cuales 595 eran varones y 371 mujeres (dos encuestados no marcaron la variable sexo), dentro de un rango de edad de 13 a 17 años y un promedio de edad de 15,27 con una desviación estándar de 1,4. Se seleccionaron tres grupos de adolescentes del siguiente modo: 437 estudiantes sobresalientes de colegios públicos y privados (con un rendimiento académico promedio de 4,2 o superior y sin problemas disciplinarios); 305 adolescentes consumidores de psicoactivos en tratamiento, de varias instituciones (comunidades terapéuticas); y 226 adolescentes contraventores de la ley penal, con medida de internamiento o de libertad vigilada emitida por un juez de infancia y adolescencia.

\section{Hallazgos}

\subsection{Clínica de una pandemia}

Plantearle a la psicología la posibilidad de aplicar el método clínico a un fenómeno social implica obligarla a ir más allá de su tradicional experticia en psicología de individuos, para recuperar una mirada que supere la especialización en objetos fragmentados: mente, comportamiento, subjetividad; $y$, ante todo, la artificial división entre objetivo-subjetivo, exterior-interior, individuo-sociedad. Así mismo, la clínica psicológica ha procurado la construcción de instrumentos para diagnosticar y evaluar, con miras a realizar intervenciones que terminan por adaptar a las personas al orden establecido y, como buena heredera de la medicina, ordenar en patologías y cuadros diagnósticos, lo que le ha permitido realizar un proceso de inclusión-exclusión dentro del marco social (Foucault, 1991, 2001; Moreno-Carmona, 2011).

No se trata solamente de considerar la realidad social como la manera subjetiva en que la perciben los individuos, sino de proponer un estudio de lo humano como una formación configurada desde lo social, un modo suyo, una resultante suya (Otero, 1999).

Es a partir de la clínica propuesta por Freud (1976, 1978, 1979), de su manera de proceder que cuestiona lo que se da por normal, que se propone como posible una clínica de lo social, problematizadora de la pretendida armonía y orden que recluye lo humano al imponerle formas masificadas y homogenizadas de ser, dejándole cada vez menos opciones de expresión de lo singular y donde el fenómeno social, se entendería como síntoma, como texto a ser develado, que anuncia malestar (Moreno-Carmona, 2011). 
Estudiar, por tanto, un fenómeno social -como la pandemia de COVID-19- es hacer la travesía por sus manifestaciones, posibles síntomas, puertas de salida a la reclusión impuesta por un modelo social centrado en el consumo y el individualismo, específicamente en la familia por cuanto irrumpen sobre aquello que se ha presupuesto como una aceptación generando desconfianza donde las cosas se asumían como dadas, mediante el reconocimiento de una especie de llamado de conciencia a la indiferencia social.

Abordar clínicamente la pandemia pone en crisis las aparentes relaciones que se venían dando y que se habían establecido como continuos, inalterables, permanentes y normales, como la dedicación al trabajo, la ausencia creciente de los padres en el proceso de crianza, la producción desenfrenada, la movilidad, las interacciones sociales, etc.

No podría mirarse la pandemia como un fenómeno aislado, sino como una resultante de la acción humana, y algunas de las posibilidades que este fenómeno ha generado tienen que ver con la creación de condiciones que se podrían leer en términos de la necesidad de replantear o incluso de crear nuevas formas de relacionarse. Tal vez lo más paradójico de esta pandemia "es que nos recuerda lo humanos que somos, que estamos vivos y que la sociedad tiene sentido en tanto compuesta por seres humanos, y no simples objetos de uso" (Moreno-Carmona, 2011 p. 7).

Resulta evidente la reclusión impuesta por la pandemia. Solo basta pensar en la limitación para salir de casa o de la ciudad, para moverse en ciertos horarios o por ciertas zonas de la ciudad, o las medidas de distanciamiento físico que limitan la expresión del afecto. Pero existe otra forma de reclusión, menos evidente, presente incluso antes de la pandemia, que es la que más interesa, pues no se trata ya de mecanismos de exclusión, propios de la patología, sino de mecanismos de reclusión, propios de lo normal (MorenoCarmona, 2011).

\subsection{Una lectura clínico social desde la dimensión del empleo}

Lejos de reducirse a una actividad remunerada, el trabajo en la modalidad de empleo da lugar a sensibilidades, pensamientos y formas de relación (Luque-Ramos, GómezDelgado y Cruces-Montes, 2000). Es decir, que el empleo es un estructurador de la vida en sociedad (Aguilar-Bustamante y Rentería-Pérez, 2009). La familia, por su parte, no es una realidad dada e inmutable. Su organización y sentir también es producto de la interacción compleja entre múltiples fenómenos sociales y económicos.

Históricamente, los arreglos familiares, es decir, quién hace qué, cómo se relaciona quién con quién, qué se prescribe y qué se proscribe, han tenido un estatuto cambiante. Una gama amplia de dimensiones de análisis que permitirían explicar estos procesos incluye el lugar del adulto como referente, el empleo, la movilidad familiar, las condiciones de 
existencia, el nivel educativo, las representaciones de género, y las nuevas tecnologías de la información y la comunicación (Moreno-Carmona, 2013).

Actualmente, la pandemia del COVID-19 ha hecho que los gobiernos nacionales implementen medidas de confinamiento. Este fenómeno ha mostrado claramente la artificialidad de las fronteras humanas, a las que la pandemia no obedece, y quizás esté indicando a la humanidad que las formas actuales de ser padres son configuradas socialmente y que, por tanto, puede haber virajes drásticos en la que pretende ser su trayectoria necesaria.

La ausencia de los padres en las casas quizás obedezca a unas lógicas del mundo laboral que, con gran esfuerzo, pueden ser revertidas. Los hallazgos de la investigación revisada reportan que -sustentada en la necesidad de trabajo, promoción académica y profesional, y en proyectos de vida personales- la educación de las familias modernas orienta en forma impersonal a sus hijos (Moreno-Carmona, 2018). Pero suponer la inevitabilidad de tal ausencia paterna quizás sirva al mantenimiento de un ordenamiento de tipo económico y político. Ahora bien, eso no significa que la presencia paterna, bajo las constelaciones actuales, sea la solución. El camino parece ser más pedregoso, pero desde estas consideraciones se pueden tener más luces sobre el malestar de algunas familias.

Bauman $(2000,2003)$ da claves para comprender cómo se configura ese ordenamiento desde su trabajo sobre la globalización, que también puede entenderse como la mundialización de la economía. La economía ha escapado al control político de los Estados individuales, de ahí que exprese que la posibilidad de agencia política de los Estados, es decir, de tomar decisiones colectivas y de llevarlas a cabo, es más una cuestión nominal. Siguiendo sus ideas, la globalización supone que una vez se multiplique la riqueza habrá más y mejores condiciones de vida para todos. Sin embargo, su análisis le indica que la globalización tiene entre sus consecuencias más notorias la concentración del capital, las riquezas y las finanzas en una minoría.

En el mundo del trabajo, y en la vía de los planteamientos de Castel (1997) se entiende que la globalización supone el fin del Estado de Bienestar, Social, o de Providencia. Este tipo de Estado se caracterizaba por procurar las transiciones que gradualmente permitieran acercarse a un ideal de progreso, y por planificar la seguridad de todos sus miembros.

Desde comienzos del milenio, en varias esferas sociales, el mundo del trabajo se rige en la lógica de la flexibilidad (Sennett, 2000), y también se observa un repliegue en el crecimiento del empleo y casi que el fin del empleo pleno. Por supuesto, las sensibilidades y formas de relación de la familia colombiana han tomado forma en este panorama que explican los sociólogos. Hay que hacer la salvedad claramente con los aportes del estudio 
de las familias colombianas, que no ocurre de la misma manera en todos las regiones y sectores sociales (Gutiérrez de Pineda, 1997, 1999; Moreno-Carmona, 2018).

En el siglo XVIII, por ejemplo, el tipo de arreglo familiar tenía que ver con una familia extensa que se organizaba y relacionaba en un contexto ajeno a la modernización, la industrialización y el urbanismo de la ciudad. Posteriormente, en la segunda mitad del siglo XX, la industrialización, la modernización y la urbanización de las sociedades colombianas suponen un viraje tremendo en el arreglo familiar. El cambio en el modo de subsistencia y la figura del empleo pleno crean buena parte de las condiciones para la privatización de la familia.

De acuerdo con Moreno-Carmona (2018) en esta familia -que podría denominarse tradicional- la principal autoridad recaía sobre el hombre. Al tiempo que la familia constituía el lugar donde desplegaba y ejercía una autoridad indiscutible, su espacio era el de la calle, los negocios, la política y el mundo del trabajo. La mujer, por su parte, asumía la procreación, las labores del hogar, el cuidado y formación moral de los hijos.

Ahora bien, el Estado de Bienestar también permitió el paso de una vida más comunitaria a una más individual y privada. Buena parte de la crianza, en adelante, no reposaría sobre el saber tradicional de la comunidad o de los abuelos. Es este panorama el que propició en buena medida la configuración de la maternidad intensiva. El niño ahora es ubicado como centro de la vida familiar y es sentido como una continuidad narcisista de los padres. Cada una de las conquistas de su desarrollo serán celebradas. Evidentemente, y como se ha tratado de mostrar hasta el momento, no se trata de que una afectividad familiar en esos términos haya existido per se, y que siempre las familias hayan funcionado del mismo modo. Las condiciones estructurales permitieron una familia nucleada, privada, con el padre al mando y la mamá al cuidado de los hijos.

Faltaba un trecho considerable para que los efectos de la globalización signaran con la flexibilidad y la precarización al mundo del trabajo y, con ello, tuviera lugar una modificación sustancial en el modo de ser familia. Además, es necesario incluir en la escena la inserción de la mujer en la educación profesional y su ingreso al mundo del trabajo calificado porque, con este hito, y a partir de los años 60, su única meta vital no sería la de ser madre, y tampoco podría serlo de manera intensiva.

Para Moreno-Carmona (2018) este hito también tiene que ver con una diversificación en la familia colombiana, que nunca ha sido una sola, pero que se ha idealizado en la forma de la familia nuclear biparental. La ganancia de independencia económica y la nueva relación de colaboración entre cónyuges también se asocia con que aquello que sostenía una relación de pareja a lo largo del tiempo ya no lo apuntalara del todo. Hay una suerte de 
Moreno-Carmona y Cleves-Valencia

retorno a la familia extensa en la medida en que ahora serán más factibles las disoluciones de las alianzas.

Las coordenadas económicas contemporáneas han dado lugar a un arreglo familiar en el que los padres no pueden -y quizás tampoco quieren- estar tanto tiempo con sus hijos, en donde las alianzas conyugales hallan las condiciones sociales para disolverse, y en que los hijos encuentran en múltiples figuras adultas, en otros espacios tangibles o virtuales, los referentes desde los cuales constituirse, en el marco de prácticas específicas (MorenoCarmona, 2018).

Por su parte, las medidas estatales de confinamiento ante la pandemia por el COVID19 interrogan al andamiaje socioeconómico, que se refleja en el mundo laboral y que moldea las sensibilidades, mentalidades y prácticas familiares. Muestran que no hay una familia única y necesaria, en primer lugar; y que lo que se teje en su interior no tiene que ver del todo con el adentro del grupo familiar, sino con el afuera del mundo socioeconómico.

Las medidas de confinamiento ponen a la familia en un tipo de encuentro dentro de la casa -que se creía imposible- y para el cual no estaba lista. No hay que ser optimista y afirmar apresuradamente que las medidas de confinamiento pueden propiciar un encuentro familiar que vaya más allá de la presencia física en un mismo espacio. Para la familia en cuya organización se valora la idea de la familia nuclear, quizás sí. Para los padres que sufren por no poder estar en casa, y para los hijos que echan de menos la presencia paterna, también. Pero es probable que en algunas familias ni siquiera haya un encuentro pese a la permanente cercanía física. Porque había un acuerdo y un modo instaurado de ser padres, esposos e hijos; del mismo modo en que se era padres, esposos e hijos en las condiciones facilitadas por el estado de bienestar y del empleo pleno.

Entonces, expresiones de malestar al interior de la familia pueden ser leídas desde esta óptica. Entendiendo, por ejemplo, que algunas madres ya no cuentan con el repertorio de la familia tradicional para ser mamás y de ese modo encontrarse con sus hijos de manera permanente. Y que quizás un hijo adolescente, por ejemplo, sienta asfixiante, extraña e invasiva la presencia materna que antes no estaba y que ahora está todo el tiempo, y para la cual construyó una red de referentes en múltiples personas y espacios de interacción (Moreno-Carmona, 2018).

Por otra parte, también es necesario considerar los fenómenos de intensificación del trabajo. A la carga excesiva de tareas y nuevos requerimientos, muchas personas han respondido en la lógica del "sí se puede", de una manera que, seguramente, no ha pasado desapercibida a los empleadores. Esta lógica del "sí se puede" es una buena posibilidad para justificar, de manera expresa o velada, el aumento de la carga laboral ahora que los individuos han mostrado su capacidad de ajustarse a las circunstancias inusitadas. Y, 
evidentemente, el mundo empresarial y económico necesita recuperarse de sus cuantiosas pérdidas. Entonces puede ocurrir que ahora los padres estén en casa, claro que sí, pero más ocupados que antes.

¿Quiere decir esto que el encuentro es imposible? No necesariamente. Simplemente hace hincapié en que, en la lógica de lo expresado hasta el momento, se requiere de condiciones socioeconómicas que faciliten ese encuentro. Y que, si se llegan a dar, tendrían que operar paulatinamente, de modo que la familia vuelva a configurar las prácticas, sensibilidades y mentalidades necesarias para un encuentro.

No se trata, por tanto, de que el rumbo de la familia marchara necesariamente hacia el no encuentro, pues eso pasa por alto las condiciones económicas del arreglo familiar que permite dilucidar una aproximación clínica de lo social. La pandemia del COVID-19, en cambio, ha puesto en jaque a un sistema económico que jalonaba a un arreglo familiar orquestado para el no-encuentro, y que depende, ante todo, de una voluntad supraestatal (Bauman, 2000, 2003).

\subsection{Una lectura clínico social desde la dimensión de la escuela}

La escuela, al igual que todos los grandes reguladores de la vida social, ha sido una institución resultante de un orden social (Tenti-Fanfani, 2007) velado en distintos planos, cuya expresión varía en el tiempo y las geografías. Si se piensa en aquellas latitudes donde el orden contemporáneo globalizante no ha hecho sentir del todo su presencia, se entiende que la función de la escuela no hay que darla por sentado, dado su carácter contingente (Helg, 2001).

Hoy por hoy no se puede concebir un proyecto de vida para niños y adolescentes sin referencia obligada a la escuela. Hace parte de los derechos consagrados, promovidos, y defendidos constitucionalmente. Pero la escuela también ha sufrido transformaciones tremendas en sus prácticas y en su función social, que han ocurrido de manera imbricada con el devenir del mundo del trabajo que queda ejemplificada con la crisis del Estado de Bienestar y el tipo de familia a que dio lugar.

Una de las consecuencias es la que encuentra Moreno-Carmona (2018) quien subraya el fenómeno de la referencialidad en la adolescencia, entendida como la búsqueda de tipos de relaciones que permiten la organización social del sujeto, en otras personas y grupos diferentes de la familia (Cardona-Rodríguez, Osorio-Tamayo y Moreno-Carmona, 2017). Pues parecía imposible que los padres estuvieran presentes, en un lugar de atención, dedicación y orientación permanente de los hijos, dadas las coordenadas socioeconómicas contemporáneas, configuradoras de sensibilidades, modos de relación familiar, y condiciones objetivas de ser padres. 
La escuela, entonces, podría ofrecer oportunidades como institución social, tratando de brindar a los estudiantes y sus familias aquello que por diferentes circunstancias no logran recibir de las demás instituciones sociales (Mejía y Muñoz, 2018, p. 191). Es decir, reasumir su lugar de orientador de los niños y adolescentes, más allá de cumplir con una función de instrucción y preparación intelectual.

Pero la historia muestra que la privatización de la familia restó terreno a la función social de la escuela (Castillo-Carbonell, Paredes-Navarro y Bou-Blanco, 2017) pues los referentes de los adolescentes, en adelante, se configuraron en su interior. Basta con recordar el lugar de los maestros como "segundos padres", autorizados para reprender de manera verbal e incluso física a los estudiantes. Con la privatización de la familia, que puso la tutela de los niños en el ámbito restringido del hogar, y con el reconocimiento y la expansión de los derechos de los niños, la escuela asumió un lugar más de instrucción que de formación.

Entonces, en lo contemporáneo, hay una demanda de retorno a dicha función social. Sólo que se trata de un retorno en otras condiciones, pues se pide a la escuela la reasunción de un lugar de formación que ya los padres no tienen cómo cumplir, pero sin las prácticas de prescripción, sanción y proscripción de antaño. Una mirada clínica a este fenómeno, que cuestiona y devela las lógicas menos evidentes, interrogaría entonces la idea de que se le pida a la escuela el reasumir su lugar de formador. Porque justamente fue el andamiaje socioeconómico el que le quitó un terreno que ella, antaño, ya tenía. Y más allá de pedirle a la escuela que se adapte a las condiciones actuales y que tenga un lugar de referente, también podría interrogarse al mundo contemporáneo en las condiciones que crea para que los padres puedan fungir como tales.

La pandemia del COVID-19, por su parte, ha dado lugar al confinamiento de todos en la casa. Y ha mostrado que buena parte de los trabajos, entre ellos la docencia, pueden llevarse a cabo desde la telepresencia mediada por las tecnologías de la información y la comunicación. Pero el recurso a la telepresencia también ha socavado el lugar del maestro como educador. Porque la intensificación de la jornada laboral en la virtualidad (Neffa, 2015) ha hecho que los maestros dediquen grandes esfuerzos al diseño de contenidos y al aprendizaje de nuevas estrategias pedagógicas, herramientas y recursos tecnológicos, abocándose más a la instrucción que a la educación. Entonces emerge la pregunta por los referentes disponibles para los adolescentes ante unos padres que aún no han desarrollado los repertorios que exige la nueva situación de confinamiento, y ante la configuración paulatina de una nueva ausencia que tiene que ver con el lugar de educador del maestro, en un panorama de salud que muestra bastantes incertidumbres. 


\subsection{La crianza confinada}

Si se asume la experiencia de la pandemia como un período crítico para muchas familias -no sólo por lo que representa en términos de riesgos para la salud de algunos de sus miembros, sino por las dificultades económicas, laborales y los cambios en las dinámicas relacionales- se hace necesario reconocer sus posibles efectos en la familia. Uno de los planteamientos de Moreno-Carmona (2018) es que las crisis forman parte inherente de la familia y cuando no son asumidas de manera asertiva por sus integrantes ésta puede fragmentarse; es decir, "en casos más extremos suele suceder que, ante un estado de crisis inesperado y no superado, el sistema se desintegra parcial o totalmente, ocasionando inesperadas consecuencias para cada integrante" (p.49).

Podría entonces pensarse, como plantea la investigación, que el confinamiento obligado por el COVID-19 puso en tensión a la familia y significó cambios drásticos en sus dinámicas, exigiendo procesos de ajuste que han obligado a sacar a flote e implementar aspectos novedosos que hasta ese momento estaban fuera de su modo habitual de funcionar. Uno de los aspectos más críticos tiene que ver con la cantidad de tiempo dedicado por los padres a sus hijos.

Como ha sido evidenciado en los últimos años, los resultados de la investigación constataron que la figura paterna dedica menos tiempo a sus hijos, siendo más crítica dicha ausencia en los adolescentes con problemas de consumo de sustancias psicoactivas; además, se indica que la figura paterna sólo dedica más tiempo cuando aún se encuentra en el núcleo familiar. Mientras que es la figura materna quien está más "pendiente" de ellos, independientemente del tipo de familia (Figuras 1 y 2).

Figura 1. Tiempo de dedicación de la figura materna según tipo de familia.

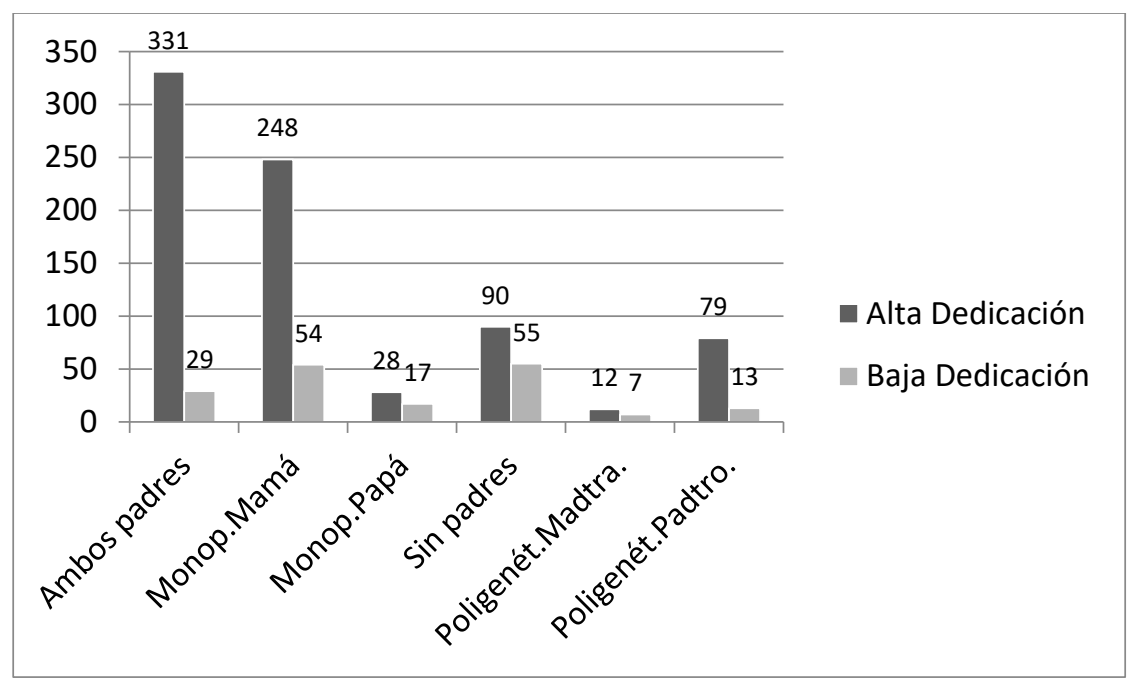

Fuente: Moreno-Carmona, 2018, p. 108. 
Figura 2. Tiempo de dedicación de la figura paterna según convivencia de los padres.

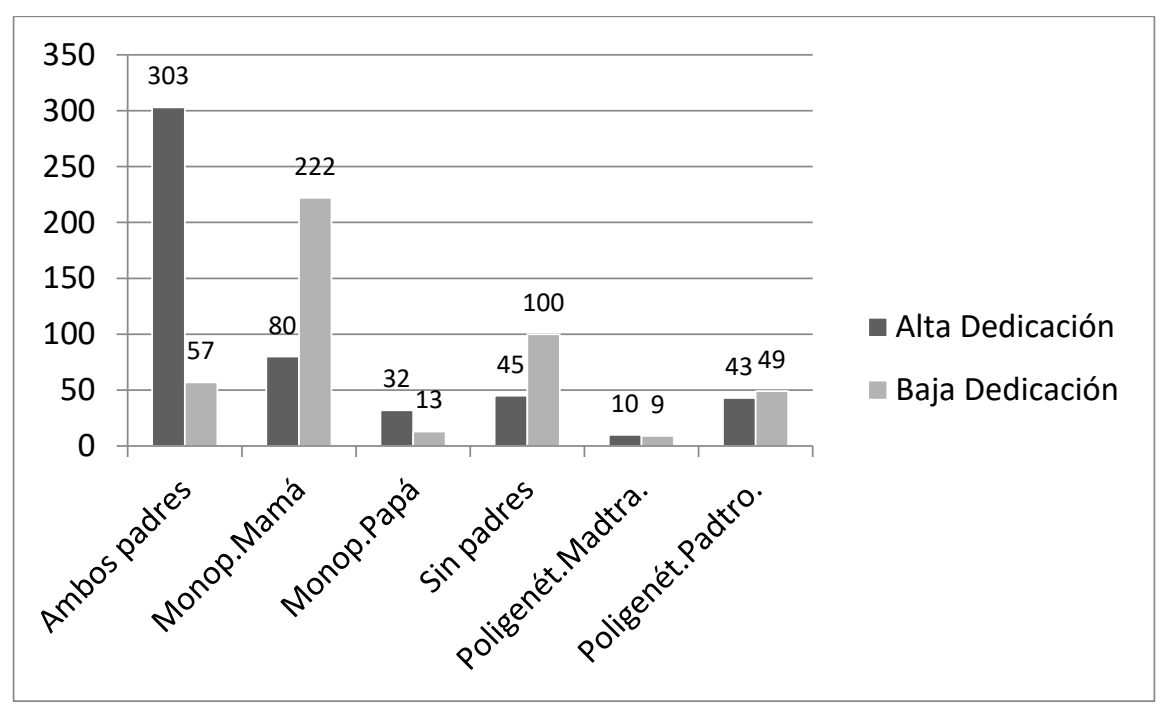

Fuente: Moreno-Carmona, 2018, p. 109

El estudio creó un índice de dedicación de tiempo de los padres mediante una variable dicotómica: dedicación alta (todo el tiempo hasta algunas horas al día) y dedicación baja (algunas horas a la semana, solo fines de semana o menos), lo que permitió identificar que los adolescentes cuyos padres les dedican menos tiempo presentan una mayor prevalencia de consumo de sustancias psicoactivas y conductas contraventoras más graves que aquellos cuyos padres están más presentes (Tabla 1).

Tabla 1. Medias, desviaciones estándar y prueba t de Student de muestras independientes para consumo de drogas y contravención según tiempo de dedicación de los padres

\begin{tabular}{|c|c|c|c|c|c|}
\hline \multirow{2}{*}{ Escala } & \multicolumn{2}{|c|}{$\begin{array}{c}\text { Alta } \\
\text { Dedicación }\end{array}$} & \multicolumn{2}{|c|}{$\begin{array}{c}\text { Baja } \\
\text { Dedicación }\end{array}$} & \multirow[b]{2}{*}{$t$} \\
\hline & $M$ & $D E$ & $M$ & $\overline{D E}$ & \\
\hline \multicolumn{6}{|l|}{ Madre } \\
\hline Prevalencia de Consumo & 6,37 & 11,54 & 9,94 & 14,5 & $-2,93^{* *}$ \\
\hline $\begin{array}{l}\text { Gravedad de } \\
\text { Contravención } \\
\text { Padre }\end{array}$ & 5,79 & 15,66 & 9,62 & 21,38 & $-2,26^{*}$ \\
\hline Prevalencia de Consumo & 5,49 & 11,41 & 8,77 & 13,24 & $-4,10^{* * *}$ \\
\hline $\begin{array}{l}\text { Gravedad de } \\
\text { Contravención }\end{array}$ & 5,23 & 16,86 & 9,99 & 16,89 & $-2,49 *$ \\
\hline
\end{tabular}


No obstante, cabe señalar que dichos hallazgos resultan poco concluyentes debido a que si bien son los estudiantes sobresalientes quienes reportan mayor tiempo de dedicación de ambos padres, también en los grupos de infractores y consumidores de sustancias psicoactivas hay un alto índice de dedicación de tiempo, tanto de la figura materna $(78,8 \%$ y $72,5 \%$ respectivamente) como de la figura paterna (31,8 \% y 43,9\% respectivamente), lo que al parecer no evitó que se involucraran en tales conductas.

Precisamente este hecho permite plantear la posible paradoja, pues, podría pensarse que las medidas de confinamiento favorecerían un mejor proceso de crianza o de interacciones paternofiliales, o que la dedicación de tiempo de los padres a los hijos permitiría una disminución en la aparición de conductas de riesgo en los niños y adolescentes.

Los datos permiten reflexionar acerca de la realidad de los procesos de crianza en la transformación familiar de las últimas décadas, es decir, la creciente ausencia de los padres por diversos motivos que ha provocado la necesidad de delegar dicha crianza en terceros o, incluso, impulsar a los hijos a desarrollar más rápidamente habilidades de independencia y autonomía. En tal sentido, un fenómeno como el confinamiento -que implicó el encierro de las familias en sus hogares- no necesariamente representa una situación ventajosa, dado que el formato de familia en el que se vive no corresponde con aquél de la preindustrialización, donde la madre ama de casa y el padre artesano participaban todo el tiempo del proceso de socialización de sus hijos.

Tal vez muchos padres se sientan abrumados con la demanda de atención permanente y muchos hijos, a su vez, saturados con la supervisión constante. La dinámica social no ha brindado herramientas para enfrentar esta situación de encierro y será necesario regular los espacios de la casa y los tiempos para permitir, a pesar de las circunstancias, ciertas distancias necesarias que sigan favoreciendo el desarrollo de la intimidad y la autonomía.

\section{Conclusiones}

Si bien los resultados de la investigación aquí revisada presentan la evidente transformación que ha sufrido la familia colombiana en las últimas décadas, también es necesario señalar que la pandemia ha dejado ver que las condiciones sociales que se daban por establecidas también son susceptibles de cambio.

Sin embargo, dichos cambios no suponen, necesariamente, mejores condiciones de vida para las familias y sus miembros. Cabe aquí reflexionar que lo primero no deslegitima lo segundo. Es decir, que hoy sea posible trabajar desde casa, o tener que participar más en los procesos de educativos y de crianza de los hijos, no representa un avance en el desarrollo positivo para los niños y adolescentes. Tampoco significa que el teletrabajo y la educación 
Moreno-Carmona y Cleves-Valencia

virtual ofrezcan mejores condiciones de empleo o de aprendizaje. Por otro lado, estas nuevas condiciones, no tan favorables, no implican que antes de la pandemia las cosas marcharan bien.

La mirada clínica que presenta esta reflexión es una invitación a interrogar las condiciones que se han venido imponiendo como normales e inevitables a propósito de instituciones como la familia, el trabajo y la escuela; a considerar el fenómeno del confinamiento como una posibilidad de pensar en alternativas diversas de trabajar, estudiar y ser familia, sin caer en la trampa de creer en un posible retorno a formas que ya no se ajustan a las nuevas condiciones de individuos y colectivos contemporáneos, y a reconocer la importancia de identificar oportunidades para el desarrollo familiar.

\section{Referencias Bibliográficas}

Aguilar-Bustamante, M. C., y Rentería-Pérez, E. (Eds.). (2009). Psicología del trabajo y de las organizaciones. Reflexiones y experiencias de investigación. Bogotá, Colombia: Editorial Universidad Santo Tomás.

Bauman, Z. (2000). Trabajo, consumismo y nuevos pobres. Barcelona, España: Gedisa.

Bauman, Z. (2003). La modernidad líquida. Buenos Aires, Argentina: Fondo de Cultura Económica.

184 Cardona-Rodríguez, F. J., Osorio-Tamayo, D. L., y Moreno-Carmona, N. D. (2017). La referencialidad, redefinida desde el bienestar subjetivo y la socialización. Revista Latinoamericana de Ciencias Sociales, Niñez y Juventud, 15(2), 1165-1177. doi: 10.11600/1692715x.1522525102016.

Castel, R. (1997). La metamorfosis de la cuestión social: una crónica del salariado. Buenos Aires, Argentina: Paidós.

Castillo-Carbonell, M., Paredes-Navarro, L., y Bou-Blanco, M. (2017). La escuela y su comunidad. La contribución de la educación social. Bogotá, Colombia: Magisterio.

Foucault, M. (1991). Enfermedad mental y personalidad. Barcelona, España: Paidós.

Foucault, M. (2001). El nacimiento de la clínica. Una arqueología de la mirada médica. México: Siglo XXI Editores.

Freud, S. (1976). Psicopatología de la vida cotidiana. En S. Freud, Obras Completas: Psicopatoiogía de la vida cotidiana (1901) (Vol. 6). Buenos Aires, Argentina: Amorrortu.

Freud, S. (1978). 17 Conferencia. El sentido de los síntomas. En S. Freud, Obras Completas. Conferencias de introducción al psicoanálisis (Parte III) - (1916-1917) (Vol. 16, pp. 235-249). Buenos Aires, Argentina: Amorrortu.

Freud, S. (1979). El malestar en la cultura. En S. Freud, Obras Completas: El porvenir de una ilusión El malestar en la cultura y otras obras (1927-1931) (Vol. 21, pp. 57-140). Buenos Aires, Argentina: Amorrortu.

Gutiérrez de Pineda, V. (1997). La familia en Colombia: trasfondo histórico. Medellín, Colombia. Editorial Universidad de Antioquia. 
Moreno-Carmona y Cleves-Valencia

Gutiérrez de Pineda, V. (1999). Estructura, función y cambio de la familia en Colombia. Medellín, Colombia. Editorial Universidad de Antioquia.

Helg, A. (2001). La educación en Colombia: 1918-1957. Una historia social, económica y política. Bogotá, Colombia: Plaza \& Janés Editores.

Luque-Ramos, P. J., Gómez-Delgado, T., y Cruces-Montes, S. J. (2000). El trabajo: fenómeno psicosocial. En R, Guil-Bozal y C, Guillén-Gestoso (Coords.), Psicología del trabajo para relaciones laborales (pp. 147-164). Bogotá, Colombia: Mc Graw Hill.

Mejía, B. M. y Muñoz, C. P. (2018). Familia y escuela, construcción de nuevos referentes. En N. D. Moreno-Carmona (Ed.), Adolescencia y Familia. La transformación de la familia colombiana y su incidencia en los adolescentes (pp. 179-191). Bogotá, Colombia: Editorial Universidad Cooperativa de Colombia.

Moreno-Carmona, N. D. (2011). Psicología del Terrorismo: el nuevo malestar en la cultura. Poiésis, (21), 1-8. doi: 10.21501/16920945.91.

Moreno-Carmona, N. D. (2013). Familias cambiantes, paternidad en crisis. Psicología desde el Caribe, 30(1), 177-209.

Moreno-Carmona, N. D. (2018). Adolescencia y Familia. La transformación de la familia colombiana y su incidencia en los adolescentes. Bogotá, Colombia: Editorial Universidad Cooperativa de Colombia.

Neffa, J. C. (2015). Los riesgos psicosociales en el trabajo: contribución a su estudio. Buenos Aires, Argentina: Centro de Estudios e Investigaciones laborales.

Otero, J. (1999). Hacia una psicología de la ciudad. Cuatro conferencias sobre clínica de lo social. Manuscrito presentado para publicación.

Sennett, R. (2000). La corrosión del carácter: las consecuencias personales del trabajo en el nuevo capitalismo. Barcelona, España: Anagrama.

Tenti-Fanfani, E. (2007). La escuela y la cuestión social: ensayos de sociología de la educación. Buenos Aires, Argentina: Siglo XXI Editores. 


\section{OTROS ARTÍCULOS DE PROSPECTIVA No. 33}

\section{EDITORIAL}

El Paro Nacional en Colombia 2021: explosión social entre dinámicas estructurales y de coyuntura. Relevancia de la acción política y del diálogo en su desarrollo y transformación Adolfo Adrián Álvarez-Rodríguez

\section{ARTÍCULOS}

Organizaciones sociales de pobladores y prácticas de resistencia en contexto de pandemia COVID-19 en Chile

Patricia Castañeda-Meneses

Entre el aislamiento y las brechas digitales: sistematización de experiencia de acompañamiento socioemocional en personas mayores de Temuco, Chile, en tiempos de COVID-19

G. Bernarda Aedo-Neira

Percepción de apoyo social y calidad de vida: la visión de personas mayores chilenas en el contexto de pandemia durante el 2020

Claudia Elena Quiroga-Sanzana

Gabriela Rocío Parra-Monje

Camila Julia Moyano-Sepúlveda

Marco Alejandro Díaz-Bravo

A propósito del qué, cómo y para qué investigar en el campo de la justicia juvenil: La trastienda de un proceso de investigación en Rosario, Argentina Karina De Bella

Proyección social: reflexión sobre una experiencia virtual con adolescentes en época de pandemia en Sucre y Bolivar, Colombia

Claudia Yaneth Martínez-Mina
La intervención social mediante proyectos comunitarios en modalidad virtual en Mérida, México. Una perspectiva desde el Trabajo Social Claudia Isabel Tzec-Puch Amairani Aracelly Ceh-Alvarado Yanet Guadalupe González-Canul

Resignificación de los vínculos Sociales durante la pandemia de COVID-19: narrativas de personas que convergen en Conjuntos residenciales en Bogotá, Colombia

Andrea Marcela Reyes-García

Laura Daniela Molina-Ortiz

La paradoja de la familia doblemente recluida: clínica de una pandemia en Colombia

Norman Darío Moreno-Carmona

Juan José Cleves-Valencia

Ciencias sociales, mundo y pandemia: por un futuro posible

José Miguel Segura-Gutiérrez

Lina Paola Vásquez-Ávila

¿Qué es Trabajo Social? Los primeros vínculos de los estudiantes con la profesión: experiencias de Argentina, Brasil, Paraguay y Uruguay

Silvia Orieta Rivero-Rodríguez

Leonel Del Prado

Nidia Graciela Battilana-Amarilla

Rosilaine Coradini-Guilherme

Intervención profesional de trabajadores sociales de la universidad del Quindio, Colombia. Asuntos epistemológicos, metodológicos y ético-políticos

Ana María Gil-Ríos 
Reflexiones sobre la intervención social en lo rural: experiencias en el Magdalena Medio, Colombia

Claudia Milena Quijano-Mejía

Johana Linares-García

Construcción de rutas de atención integral a la convivencia escolar en Cali, Colombia: sistematización de la experiencia

Leidy Johana Prado-Montaño

Luz Helena López-Rodríguez

Alejandra Gutiérrez-Cárdenas

Experiencia del proceso de intervención familiar de los actores participantes en la Escuela para Familias del ICBF-Jamundí, Colombia Katherin Viviana Silva-Minotta

María José Forero-Izquierdo

Jimena del Pilar Jaramillo-Jaramillo

\section{RESEÑAS DE LIBROS}

Alerta global: políticas, movimientos sociales y futuros en disputa en tiempos de pandemia Jairo Crispín

El neoliberalismo como teología política. Habermas, Foucault, Dardot, Laval y la historia del capitalismo contemporáneo

José Francisco Desentis-Torres

La Reconceptualización del Trabajo Social en Colombia: Análisis histórico-crítico de las décadas de 1960-1970

Juan Pablo Sierra-Tapiro

ARTISTA INVITADO

Juan Camilo González

Prospectiva

\section{PROSPECTIVA}

Revista de Trabajo Social e Intervención Social

No. 33 • ene.-jun. 2022

e-ISSN: 2389-993X • Universidad del Valle 